\title{
Mutational and expressional analyses of STAG2 gene in solid cancers
}

\author{
M. S. KIM"1, , S. S. KIM", , E. M. JE', N. J. YOO ${ }^{1}$, S. H. LEE ${ }^{1,3, *}$ \\ ${ }^{1}$ Department of Pathology, College of Medicine, The Catholic University of Korea, Seoul, Korea; ${ }^{2}$ Department of Internal Medicine, College \\ of Medicine, The Catholic University of Korea, Seoul, Korea; ${ }^{3}$ Research Center for Genome Polymorphism, College of Medicine, The Catholic \\ University of Korea, Seoul 137-701, Korea
}

${ }^{*}$ Correspondence: suhulee@catholic.ac.kr

Contributed equally to this work.

Received February 2, 2012 / Accepted March 23, 2012

\begin{abstract}
Aneuploidy is frequently observed in cancers and is considered a crucial mechanism in cancer development. STAG2 is a gene that encodes a component of cohesion complex required for normal chromosomal segregation. Recently, somatic mutation of STAG2 gene and loss of STAG2 protein have been reported in glioblastoma, Ewing's sarcoma and melanoma. The aim of this study was to see whether such alterations of STAG2 are also common in other cancers. In this study, we analyzed STAG2 somatic mutation in 45 colorectal carcinomas (CRC), 45 gastric carcinomas (GC), 45 breast carcinomas, 45 non-small cell lung cancers and 45 prostate carcinomas (PCA) by single-strand conformation polymorphism. We analyzed also STAG2 protein expression in 100 GC, 103 CRC and 107 PCA by immunohistochemistry. STAG2 protein was well expressed in normal stomach, colon and prostate epithelial cells, while it was lost in 27\% of GC, 23\% of CRC and $30 \%$ of PCA. The loss of STAG2 was observed irrespective of subtypes, stages and grades of the cancers. However, we could not find any STAG2 mutations in these cancers. The loss of expression of STAG2 in GC, CRC and PCA tissues compared to their corresponding normal cells indicates that STAG2 loss is common in carcinomas as well. The data suggest also that loss of expression of STAG2, but not somatic mutation, might be responsible to STAG2 inactivation and is common in studied types of carcinomas.
\end{abstract}

Key words: STAG2, aneuploidy, expression, mutation, gastric cancer, colorectal cancer, prostate cancer

Aneuploidy, a type of chromosome abnormality, is an abnormal number of chromosomes that occurs during cell division when the chromosomes do not separate properly between the two cells [1]. Aneuploidy is a common cause of genetic disorders and is frequently observed in cancers (in more than $90 \%$ of human solid cancers) [1-5]. However, it has not been clear that aneuploidy is a cause or a consequence of carcinogenesis. Correlation of specific aneuploidies with distinct cancer phenotypes, elevated chromosomal instability in aneuploid cancer cells and presence of mutations in genes regulating chromosome segregation have suggested that aneuploidy may be a cause of cancers [6-13].

Recently, Solomon et al. discovered somatic mutations in STAG2 gene encoding a subunit of cohesin complex that regulates separation of sister chromatids during cell division [14]. In the cell lines with a stable karyotype a targeted inactivation of STAG2 gene led to chromatid cohesin defects and aneuploidy, while in the aneuploid cell lines targeted correction of the endogenous mutant alleles of STAG2 gene led to an enhanced chromosomal stability [14]. The STAG2 mutations were found in glioblastomas, melanomas and Ewing's sarcomas, and consisted of truncating mutations, missense mutations, splicing-site mutations and intragenic deletions [14]. Also, loss of STAG2 protein expression was observed in glioblastomas, Ewing's sarcomas, melanomas, lymphomas, medulloblastomas and colorectal carcinomas in a range of 3-21\% depending on the cancer types [14]. These data indicate that expression loss and somatic mutation of STAG2 occur in human cancers, and suggest that inactivation of STAG2 may contribute to tumorigenesis by causing aneuploidy in the cells.

Although loss of STAG2 protein and somatic mutation of STAG2 gene have been identified, most of them have been studied in cancers with non-epithelial origins [14]. In the present study, we analyzed somatic mutation of STAG2 gene and expression of STAG2 protein in several human carcinomas. 


\section{Materials and Methods}

Polymerase chain reaction (PCR) and single-strand conformation polymorphism (SSCP). For the mutation analysis of STAG2, methacarn-fixed and paraffin-embedded tissues of 45 colorectal carcinomas (CRC), 45 gastric carcinomas (GC), 45 breast carcinomas, 45 non-small cell lung cancers and 45 prostate carcinomas (PCA) were randomly selected for the study. The CRC originated from cecum $(\mathrm{N}=1)$, ascending colon $(\mathrm{N}=7)$, transverse colon $(\mathrm{N}=2)$, descending colon $(\mathrm{N}=2)$, sigmoid colon $(\mathrm{N}=13)$ and rectum $(\mathrm{N}=20)$. The GC consisted of 22 diffuse-type, 17 intestinal-type and six mixed-type GC by Lauren's classification, and 45 advanced GC according to the depth of invasion. The non-small cell lung cancers consisted of 24 adenocarcinomas and 21 squamous cell carcinomas. The breast carcinomas consisted of 45 invasive ductal carcinomas (10 grade I, 17 grade II and 18 grade III). All of the patients were Asians (Korean). Approval was obtained from the Catholic University of Korea, College of Medicine's institutional review board for this study. For the solid tumors, tumor cells and normal cells from the same patients were selectively procured from hematoxylin and eosin-stained slides using a 30G1/2 hypodermic needle by the microdissection. DNA extraction was performed by a modified single-step DNA extraction method by proteinase $\mathrm{K}$ treatment.

Coding sequence of STAG2 gene (NM_001042750) consists of 33 coding exons (exons 3-35) that translate 1268 amino acids. In the previous report, a vast majority $(83 \%$ $(10 / 12))$ of STAG2 mutations in the coding region and splice-sites were found in exons 9, 11, 12 and 20 [14]. Thus, we analyzed these exons by PCR-SSCP. Genomic DNA each from tumor cells and normal cells from the same patients were amplified with five primer pairs covering these exons of human STAG2 gene. Radioisotope $\left(\left[{ }^{32} \mathrm{P}\right] \mathrm{dCTP}\right)$ was incorporated into the PCR products for detection by autoradiogram. After amplification, PCR products were denatured and were loaded onto SSCP gel (Cambrex Bio Science Rockland, Rockland, ME). Other procedures of the PCR-SSCP were described in our previous studies $[15,16]$. Mobility shifts on SSCP were determined by visual inspection. Sequencing of the PCR products was carried out using a capillary automatic sequencer (3730 DNA Analyzer, Applied Biosystem, Carlsbad, CA). To confirm the SSCP data, we repeated the PCR-SSCP twice.

Tissue microarray (TMA). Overall, 11 TMA recipient blocks were used in this study for immunohistochemisty of STAG2. The TMA contained paraffin-embedded PCA (four blocks), GC (six blocks) and CRC (one block) tissues from archival patient specimens, previously fixed in $10 \%$ formaldehyde. The CRC $(\mathrm{N}=103)$ originated from cecum $(\mathrm{N}=2)$, ascending colon $(\mathrm{N}=19)$, transverse colon $(\mathrm{N}=6)$, descending colon $(\mathrm{N}=4)$, sigmoid colon $(\mathrm{N}=28)$ and rectum $(\mathrm{N}=44)$. TNM stages of the CRC were $35 \mathrm{I}, 30 \mathrm{II}, 31 \mathrm{III}$ and seven IV. From each donor CRC block, four cylinders (one from normal and three from cancer tissues) of $1.0-\mathrm{mm} \mathrm{di}$ ameter tissue were taken from representative areas. Ages of the patients ranged from 29-80 years with an average of 53.6 years. The GC $(\mathrm{N}=100)$ consisted of 50 diffuse, 36 intestinal and 14 mixed-type GC by Lauren's classification, and 4 early GC and 96 advanced GC according to the depth of invasion. TNM stages of the GC were four IA, 15 IB, 79 II and two IIIA. From each donor GC block, two cylinders (one from nor$\mathrm{mal}$ and one from cancer tissues of each patient) of $2.0-\mathrm{mm}$ diameter tissue were taken. Ages of the GC patients ranged 29-89 years with an average of 60 years. The PCA $(\mathrm{N}=107)$ consisted of one Gleason score 5, 10 score 6, 47 score 7, 10 score 8 and 39 score 9 cancers. Ages of the PCA patients ranged 43-77 years with an average of 67.6 years. Sizes of the cancers ranged $1.1-5.0 \mathrm{~cm}$ in diameter with an average of 2.5 $\mathrm{cm}$. From every archival paraffin block, five cylinders (two from normal and three from cancer tissues of each patient: total 535 cylinders) of $1.0-\mathrm{mm}$ diameter tissue were taken from representative areas.

Using sections from the TMA, immunohistochemistries for STAG2 were performed using DAKO REAL EnVision System (DAKO, Glostrup, Denmark) with a mouse monoclonal antibody against human STAG2 (Santa Cruz Biotechnology, Santa Cruz, USA; dilution 1/50). After deparaffinization, heat-induced epitope retrieval was conducted by immersing the slides in Coplin jars filled with $10 \mathrm{mmol} / \mathrm{L}$ citrate buffer (pH 6.0) and boiling the buffer for $30 \mathrm{~min}$ in a pressure cooker inside a microwave oven at $700 \mathrm{~W}$. Sections were incubated overnight at $4^{\circ} \mathrm{C}$ with the primary antibody. The reaction products were developed with diaminobenzidine and counterstained with hematoxylin. Other procedures were described in our previous studies [17]. Tumors were interpreted as positive by immunohistochemistry when nuclear staining was seen in greater than $30 \%$ of the neoplastic cells. The results were reviewed independently by two pathologists. As negative controls, a slide was treated by replacement of primary antibody with the blocking reagent. For the statistical analysis of the data, we used Fisher's exact test and $\chi^{2}$ test.

\section{Results}

Genomic DNAs isolated from the 45 CRC, 45 GC, 45 breast carcinomas, 45 non-small cell lung cancers and 45 PCA tissues were analyzed for detection of somatic mutations in STAG2 gene by PCR-SSCP analysis. On the SSCP autoradiograms, all of the PCR products were clearly seen. However, none of the SSCP from the microdissected cancer cells revealed aberrantly migrating bands compared to wild-type bands from normal tissues, indicating there was no evidence of STAG2 mutation in the cancers analyzed (Figure 1). To confirm the SSCP data, we repeated the experiments twice, including tissue microdissection, PCR and SSCP to ensure specificity of the results, and found that the data were consistent.

With the immunohistochemical approach using TMA, we analyzed expression of STAG2 in normal and tumor 


\section{STAG2 Exon 20}

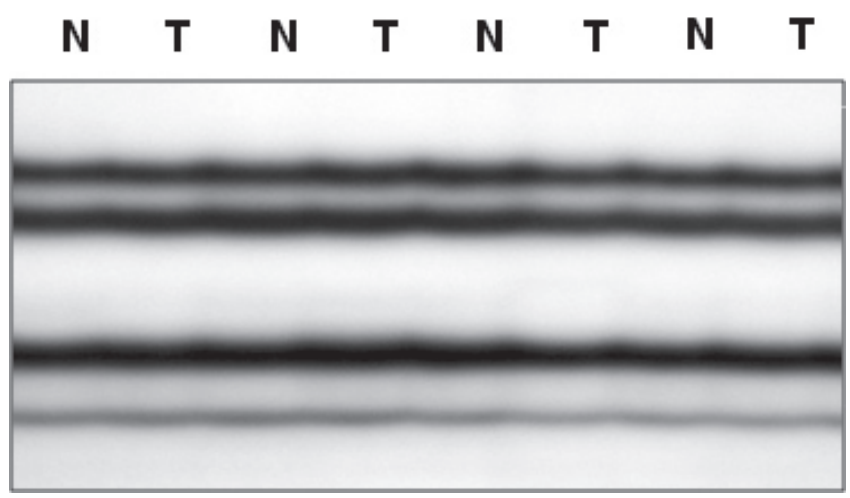

Figure 1. Representative SSCP of STAG2 gene. Exon 20 of STAG2 gene (A) in 5 different $\mathrm{GC}$ was amplified using a specific primer set. There is no aberrant SSCP band in cancer tissues $(\mathrm{T})$ compared to normal tissues $(\mathrm{N})$.

tissues from GC, CRC and PCA patients. The data of the immunostainings are summarized in Table 1. There was no variation of staining between the cores from the same tumors. In the GC, immunopositivity for STAG2 was observed in $73(73.0 \%)$ of the 100 cancers (Figure 2A and B). According to the Lauren's subtypes, STAG2 immunostaining was similarly detected in the GC irrespective of the subtypes (77.8\% of the intestinal-type GC, $72.0 \%$ of the diffuse-type GC and $64.3 \%$ of the mixed-type GC). By contrast, normal mucosal epithelial cells (surface mucosal cells, mucosal glandular cells and fundic gland cells) showed STAG2 immunopositivity in all of the cases (Table 1, Figure 2C). In the CRC, immunopositivity for STAG2 was observed in $79(76.7 \%)$ of the 103 cancers (Figure 2D and E). Normal colonic mucosal epithelial cells showed STAG2 immunoreactivity in $100 \%$ of the cases (Figure $2 \mathrm{D}$ and F). In the PCA, immunopositivity was observed for STAG2 in 75 (70.1\%) of the 107 cancers (Figure $2 \mathrm{G}$ and $\mathrm{H}$ ). In normal prostate glands, STAG2 expression was observed in both basal and

Table 1. Summary of the STAG2 expression in gastric, colorectal and prostate tissues

\begin{tabular}{llcc}
\hline Organs & tissues & $\begin{array}{c}\text { Total } \\
\text { No. }\end{array}$ & $\begin{array}{c}\text { No. positive } \\
(\%)\end{array}$ \\
\hline Stomach & Normal gastric mucosal epithelial cells & 100 & $100(100)$ \\
& Gastric adenocarcinomas & 100 & $73(73.0)$ \\
& - Intestinal type & 36 & $28(77.8)$ \\
& - Diffuse type & 50 & $36(72.0)$ \\
& - Mixed type & 14 & $9(64.3)$ \\
\hline Colon & Normal colonic mucosal epithelial cells & 103 & $103(100)$ \\
& Colorectal adenocarcinomas & 103 & $79(76.7)$ \\
\hline Prostate & Normal basal cells & 107 & $107(100)$ \\
& Normal alveolar cells & 107 & $107(100)$ \\
& Prostate adenocarcinomas & 107 & $75(70.1)$ \\
\hline
\end{tabular}

alveolar cells (Figure 2G and I). The STAG2 immunostaining, when present, was found in the nuclei of the cells, but negative or very weak in the cytoplasm (Figure 2A, C, D, F, G and I). Negative control using the blocking solution instead of the primary antibody showed no signal (Figure 2J). For STAG2 negative samples by immunohistochemistry, we tested other antibodies and found that they were accessible to them (data not shown).

Statistically, there were differences of STAG2 immunostaining between normal and GC (Fisher's exact test, $p<$ 0.001 ), normal and CRC (Fisher's exact test, $p<0.001$ ), and normal and PCA (Fisher's exact test, $p<0.001$ ). There was no statistical difference of STAG2 immunostaining among GC, CRC and PCA (Fisher's exact test, $p>0.05$ ). There was not any difference of the STAG2 expression with respect to Lauren's subtypes in GC, TNM stages of GC, TNM stage of CRC and Gleason grades in PCA (Fisher's exact test, $p>0.05)$. Next, we analyzed the relationship of the immunostaining with various pathologic parameters (age, sex, and depth of invasion). However, there was no significant association ( $\chi^{2}$ test, $p>0.05$ ).

\section{Discussion}

Frequent observation of aneuploidy in human cancer cells and its causal implication in tumorigenesis [6-10] led us to analyze somatic mutation and expression of STAG2 gene, mechanisms involved in aneuplidy production in cancer cells, in GC, CRC and PCA tissues. In the present study, the data showed that STAG2 protein was well expressed in normal gastric, colonic and prostate epithelial cells. However, its expression was lost in $27 \%$ of gastric, $23 \%$ of colorectal and $30 \%$ of prostate cancers. Of note, the STAG2 loss was observed in these cancers irrespective of TNM stages and histologic subtypes. By contrast, we found no STAG2 somatic mutations in GC, CRC, PCA, breast cancers and lung cancers. Together, these data indicate that loss of STAG2 expression, but not somatic mutation of STAG gene, might be a feature of common solid cancers, and suggest that such loss of STAG2 expression might contribute to their pathogenesis by producing aneuploidy in cancer cells.

To our knowledge, there has been only one immunohistochemical study on STAG2 expression in human cancers [14]. In that study, loss of STAG2 expression was observed in $21 \%$ of Ewing's sarcomas, $19 \%$ of glioblastomas, $19 \%$ of melanomas, $5 \%$ of medulloblastomas, $3 \%$ of colorectal cancers and $2 \%$ of lymphomas. Together, our data and the previous data show that loss of STAG2 expression is widespread in human cancers, and is present in carcinomas as well as in non-carcinomatous malignancies. However, there is a significantly difference of STAG2 loss in colorectal cancers (Fisher's exact test, $p<0.001)$ between our study $(21 \%)$ and the earlier study $(2 \%)$. Whether this discrepancy is originated from ethnic difference or other factors remains to be identified in further studies. 

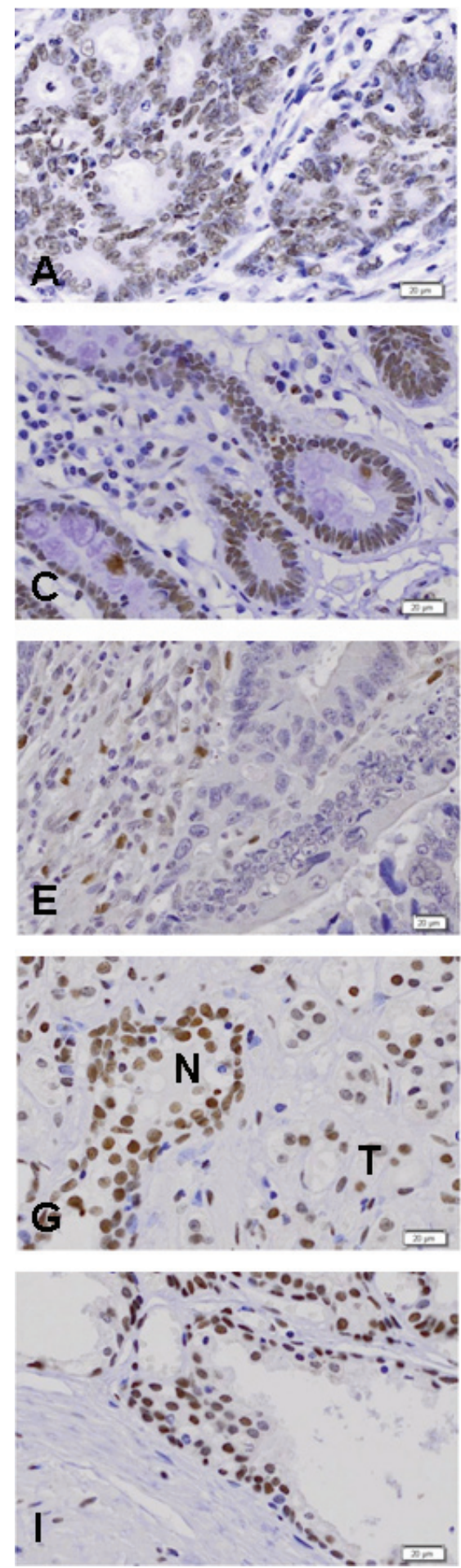
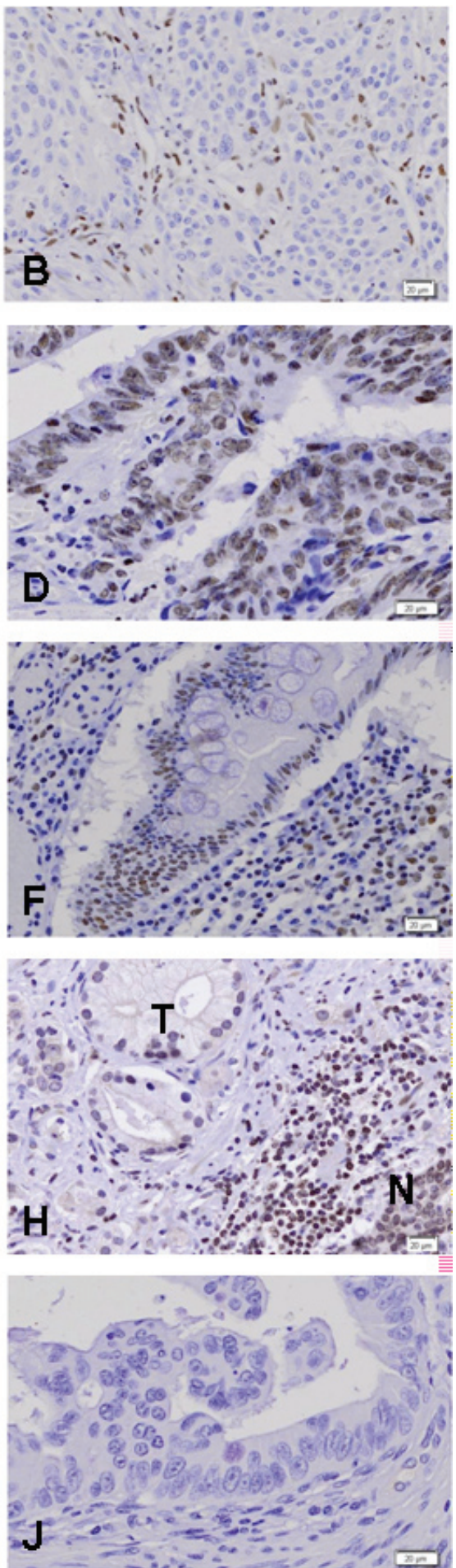

Figure 2. Visualization of STAG2 expression in gastric, colorectal and prostate cancer tissues by immunohistochemistry. A: A gastric adenocarcinoma shows a positive immunoreactivity for STAG2. B: In another gastric adenocarcinoma, the cancer cells are negative for STAG2 immunostaining. C: Normal gastric mucosal epithelial cells are positive for STAG2 immunostaining. D: A colon adenocarcinoma (T) shows a positive immunoreactivity for STAG2. E: In another colon adenocarcinoma, the cancer cells are negative for STAG2 immunostaining. F: In a normal mucosal epithelium of colon, the cells are positive for STAG2 immunostaining. G: A prostate adenocarcinoma shows immunoreactivity for STAG2 in the cancer cells (T). Adjacent normal prostate gland cells show immunoreactivity for STAG2 (N). H: In another prostate adenocarcinoma, the cancer cells are negative for STAG2 immunostaining. Adjacent normal prostate gland cells show immunoreactivity for STAG2 (N). I: In normal prostate glands, both basal and alveolar cells are positive for STAG2 immunostaining. J: A negative control of the immunostaining with omission of primary antibody shows no STAG2 immunostaining. (Scale bars: 20 um) 
The previous study identified STAG2 somatic mutations in $10.1 \%$ (7/69) of glioblastomas, $29.2 \%$ (7/24) of Ewing's sarcomas and $4.2 \%(2 / 48)$ of melanomas (14). However, we were not able to find STAG2 somatic mutations in 315 cancers from CRC, GC, breast carcinomas, non-small cell lung cancers and PCA. These data could be interpreted several ways. First, STAG2 mutation may be specific to glioblastoma, Ewing's sarcoma and melanomas, while it may be rare in other site of solid cancers analyzed in the present study. Further studies are needed to find STAG2 mutations in other cancers, including various sarcomas. In the previous study [14], most of the STAG2 mutations (10 of the total 16 mutations) were detected in cancer cell lines. A possible difference in STAG2 mutation between cell lines and primary tissues need to be clarified as well. Under a suitable condition, SSCP is capable of detecting over $90 \%$ of mutations within any sequence, and the sensitivity is generally believed to be high if the amplified fragments are 200 bps or less in size [18]. It is possible that some mutational changes may not be detected by SSCP in this study. However, since we have analyzed the samples by SSCP twice times and used primers around $200 \mathrm{bp}$, it can be thought that the missing of STAG2 mutations were very rare in this study.

In our study, we failed to find any relationship of STAG2 expression with available clinicopathologic parameters, including TNM stage and Gleason score. STAG2 expression was observed evenly through low to high TNM stages in GC and CRC, and low to high Gleason scores in PCA. This might be because loss of STAG2 expression might occur at early stage of the cancer development.

Deletion of chromosome Xq25, where STAG2 is mapped, is occasionally observed in some cancers, including ovarian cancer, AML and glioblastoma [14, 19-21]. In addition, somatic mutation of STAG2 gene was identified as a main cause of STAG2 loss in glioblastoma, Ewing's sarcoma and melanoma [14]. In our study, we analyzed somatic mutation of STAG2, a possible cause of STAG2 loss, but could not find any of STAG2 mutations. Although we did not analyze deletion of Xq25 in the present study, it doesn't seem that deletion of Xq25 can fully explain the frequent loss of STAG2 expression in the cancers analyzed in this study, because deletion of Xq25 is not known to be common in these cancers [22]. Other gene silencing mechanisms such as promoter methylation and transcriptional downregulation might contribute to of STAG2 loss in these cancers.

In summary, the present study discovered that STAG2 expression is lost in about one fourth of gastric, colorectal and prostate cancers compared to their normal cells, suggesting its contribution to their tumorigenesis. To see whether loss of STAG2 expression is a general mechanism that medicates aneuploidy development in human cancers, it may be necessary to analyze expressional alterations of STAG2 together with somatic mutation in other cancers.

Acknowledgements: This study was supported by a grant from Ministry for Health, Welfare and Family Affairs (A111513).

\section{References}

[1] TORRES EM, WILLIAMS BR, AMON A. Aneuploidy: cells losing their balance.Genetics 2008; 179: 737-746.

[2] HASSOLD TJ, JACOBS PA. Trisomy in man. Annu Rev Genet 1984; 18: 69-97.

[3] WILLIAMS BR, AMON A. Aneuploidy: cancer's fatal flaw? Cancer Res 2009; 69: 5289-5291. http://dx.doi.org/10.1158/ 0008-5472.CAN-09-0944

[4] WEAVER BA, CLEVELAND DW. Aneuploidy: instigator and inhibitor of tumorigenesis. Cancer Res 2007; 67: 10103-10105. http://dx.doi.org/10.1158/0008-5472.CAN-07-2266

[5] HOLLAND AJ, CLEVELAND DW. Boveri revisited: chromosomal instability, aneuploidy and tumorigenesis. Nat Rev Mol Cell Biol 2009; 10: 478-487. http://dx.doi.org/10.1038/ $\underline{\text { nrm2718 }}$

[6] KRAUTER J, GANSER A, BERGMANN L, RAGHAVACHAR A, HOELZER D et al. Association between structural and numerical chromosomal aberrations in acute myeloblastic leukemia: a study by RT-PCR and FISH in 447 patients with de-novo AML. Ann Hematol 1999; 78: 265-269. http://dx.doi. org/10.1007/s002770050512

[7] VAN DEN NESTE E, LOUVIAUX I, MICHAUX JL, DELANNOY A, MICHAUX L et al. Myelodysplastic syndrome with monosomy 5 and/or 7 following therapy with 2-chloro2'-deoxyadenosine. Br J Haematol 1999; 105: 268-270. http://dx.doi.org/10.1111/j.1365-2141.1999.01277.x

[8] LENGAUER C, KINZLER KW, VOGELSTEIN B. Genetic instability in colorectal cancers. Nature 1997; 386: 623-627. http://dx.doi.org/10.1038/386623a0

[9] JIN DY, SPENCER F, JEANG KT. Human T cell leukemia virus type 1 oncoprotein Tax targets the human mitotic checkpoint protein MAD1. Cell 1998; 93: 81-91. http://dx.doi. org/10.1016/S0092-8674(00)81148-4

[10] ZHOU H, KUANG J, ZHONG L, KUO WL, GRAY JW et al. Tumour amplified kinase STK15/BTAK induces centrosome amplification, aneuploidy and transformation. Nat Genet 1998; 20: 189-193. http://dx.doi.org/10.1038/2496

[11] ZOU H, MCGARRY TJ, BERNAL T, KIRSCHNER MW. Identification of a vertebrate sister-chromatid separation inhibitor involved in transformation and tumorigenesis. Science 1999; 285: 418-422. http://dx.doi. org/10.1126/science.285.5426.418

[12] TORRES EM, DEPHOURE N, PANNEERSELVAM A, TUCKER CM, WHITTAKER CA et al. Identification of aneuploidy-tolerating mutations. Cell 2010; 143: 71-83. http://dx.doi.org/10.1016/j.cell.2010.08.038

[13] SHELTZER JM, BLANK HM, PFAU SJ, TANGE Y, GEORGE $\mathrm{BM}$ et al. Aneuploidy drives genomic instability in yeast. Science 2011; 333: 1026-1030. http://dx.doi.org/10.1126/ science. 1206412

[14] SOLOMON DA, KIM T, DIAZ-MARTINEZ LA, FAIR J, ELKAHLOUN AG et al. Mutational inactivation of STAG2 causes aneuploidy in human cancer. Science 2011; 333: 1039-1043. http://dx.doi.org/10.1126/science.1203619

[15] LEE JW, SOUNG YH, KIM SY, LEE HW, PARK WS et al. PIK3CA gene is frequently mutated in breast carcinomas and 
hepatocellular carcinomas. Oncogene 2005; 24: 1477-1480. http://dx.doi.org/10.1038/sj.onc.1208304

[16] LEE SH, SHIN MS, PARK WS, KIM SY, KIM HS et al. Alterations of Fas (Apo-1/CD95) gene in non-small cell lung cancer. Oncogene 1999; 18: 3754-3760. http://dx.doi.org/10.1038/ sj.onc. 1202769

[17] KIM SY, OH YL, KIM KM, JEONG EG, KIM MS et al. Decreased expression of Bax-interacting factor-1 (Bif-1) in invasive urinary bladder and gallbladder cancers. Pathology 2008; 40: 553-557. http://dx.doi.org/10.1080/00313020802320440

[18] HAYASHI K. PCR-SSCP: A simple and sensitive method for detection of mutations in the genomic DNA. PCR Methods Appl 1992; 1: 34-38.

[19] WALTER MJ, PAYTON JE, RIES RE, SHANNON WD, DESHMUKH $\mathrm{H}$ et al. Acquired copy number alterations in adult acute myeloid leukemia genomes. Proc Natl Acad Sci
U S A 2009; 106: 12950-12955. http://dx.doi.org/10.1073/ pnas.0903091106

[20] GORRINGE KL, RAMAKRISHNA M, WILLIAMS LH, SRIDHAR A, BOYLE SE et al. Are there any more ovarian tumor suppressor genes? A new perspective using ultra highresolution copy number and loss of heterozygosity analysis. Genes Chromosomes Cancer 2009; 48: 931-942. http://dx.doi. org/10.1002/gcc.20694

[21] ROCQUAIN J, GELSI-BOYER V, ADELAIDE J, MURATI A, CARBUCCIA $\mathrm{N}$ et al. Alteration of cohesin genes in myeloid diseases. Am J Hematol 2010; 85: 717-719. http://dx.doi. org/10.1002/ajh.21798

[22] MITELMAN F, MERTENS F, JOHANSSON B. A breakpoint map of recurrent chromosomal rearrangements in human neoplasia. Nat Genet 1997; 15 Spec No:417-474. http://dx.doi. org/10.1038/ng0497supp-417 